\title{
With the greatest care, stromal interaction molecule (STIM) proteins verify what skeletal muscle is doing
}

\author{
Chung-Hyun Cho ${ }^{1}$, Keon Jin Lee ${ }^{2,3,+}$ \& Eun Hui Lee ${ }^{2,3, *}$ \\ ${ }^{1}$ Department of Pharmacology, College of Medicine, Seoul National University, Seoul 08826, ${ }^{2}$ Department of Physiology, College of \\ Medicine, The Catholic University of Korea, ${ }^{3}$ Department of Biomedicine \& Health Sciences, Graduate School, The Catholic University of \\ Korea, Seoul 06591, Korea
}

Skeletal muscle contracts or relaxes to maintain the body position and locomotion. For the contraction and relaxation of skeletal muscle, $\mathrm{Ca}^{2+}$ in the cytosol of skeletal muscle fibers acts as a switch to tum on and off a series of contractile proteins. The cytosolic $\mathrm{Ca}^{2+}$ level in skeletal muscle fibers is governed mainly by movements of $\mathrm{Ca}^{2+}$ between the cytosol and the sarcoplasmic reticulum (SR). Store-operated $\mathrm{Ca}^{2+}$ entry (SOCE), $\mathrm{a} \mathrm{Ca}^{2+}$ entryway from the extracellular space to the cytosol, has gained a significant amount of attention from muscle physiologists. Orai 1 and stromal interaction molecule 1 (STIM1) are the main protein identities of SOCE. This mini-review focuses on the roles of STIM proteins and SOCE in the physiological and pathophysiological functions of skeletal muscle and in their correlations with recently identified proteins, as well as historical proteins that are known to mediate skeletal muscle function. [BMB Reports 2018; 51(8): 378-387]

\section{INTRODUCTION}

Skeletal muscle makes up 36 to $42 \%$ of human body mass (the percentage of skeletal muscle in the body depends on gender, age, and various environmental factors), and its main task is to maintain the position of the body and locomotion via contraction and relaxation (1). Skeletal muscle contraction is achieved via excitation-contraction (EC) coupling, and $\mathrm{Ca}^{2+}$ ions from the sarcoplasmic reticulum $(S R$, an intracellular

*Corresponding author. Tel: +82-2-2258-7279; Fax: +82-2-5329575; E-mail: ehui@catholic.ac.kr

${ }^{\dagger}$ Current address: New Drug Development Center, Osong Medical Innovation Foundation, Cheongju 28160, Korea

https://doi.org/10.5483/BMBRep.2018.51.8.128

\section{Received 11 April 2018}

Keywords: Dihydropyridine receptors (DHPR), Excitation-contraction (EC) coupling, Sarcoplasmic/endoplasmic reticulum $\mathrm{Ca}^{2+}$ ATPase la (SERCA1a), Store-operated $\mathrm{Ca}^{2+}$ entry (SOCE), Stromal interaction molecule (STIM) organelle corresponding to the endoplasmic reticulum (ER) in other types of cells) work as a switch to activate a series of contractile proteins during skeletal muscle contraction (2-5). The initiation of skeletal muscle contraction is different from maintenance (i.e., the duration of the skeletal muscle contraction with a high cytosolic $\mathrm{Ca}^{2+}$ level, and is considered one of the significant parameters of skeletal muscle strength). When elucidating the maintenance of skeletal muscle contraction, skeletal muscle physiologists study classical EC coupling and pay significant attention to extracellular $\mathrm{Ca}^{2+}$ entry (6-8). One of the avenues for extracellular $\mathrm{Ca}^{2+}$ entry in skeletal muscle fibers is store-operated $\mathrm{Ca}^{2+}$ entry (SOCE) (6, 7). Orai1 (a $\mathrm{Ca}^{2+}$ entry channel protein) and stromal interaction molecule 1 (STIM1, a $\mathrm{Ca}^{2+}$ sensor protein) form a unit that is the main mediator in skeletal SOCE, as well as in other types of cells (9-18). This mini-review focuses on the roles of STIMs and SOCE in the physiological or pathophysiological functions of skeletal muscle and on the correlations with recently identified proteins, as well as historical EC coupling-mediating proteins.

\section{SKELETAL EC COUPLING}

In skeletal muscle fibers during EC coupling (2-5), acetylcholine receptors, i.e., ligand-gated $\mathrm{Na}^{+}$channels, in the sarcolemmal membrane (corresponding to the plasma membrane in other types of cells) are activated by acetylcholine that is released from motor neurons (skeletal muscle fibers are often called 'myotubes' in in vitro culture conditions). $\mathrm{Na}^{+}$influx into the cytosol through acetylcholine receptors induces depolarization of the sarcolemmal membrane ('excitation' of the skeletal muscle fibers). The spreading of the membrane depolarization to the interior of the skeletal muscle fibers via invagination of the sarcolemmal membrane (transverse (t)-tubules) activates dihydropyridine receptors (DHPRs) that is a voltage-gated $\mathrm{Ca}^{2+}$ channel on the t-tubule membrane. T-tubule membranes are closely juxtaposed with the SR membrane, which is more specialized than the ER in storing a massive amount of $\mathrm{Ca}^{2+}$ ions $(3,4,19-22)$. Collectively, this membrane complex is referred to, either as the triad junction, or as the junctional membrane complex. Physical stimulation 
induces the active DHRP to activate ryanodine receptor 1 (RyR1), which is a ligand-gated $\mathrm{Ca}^{2+}$ channel on the SR membrane. $\mathrm{Ca}^{2+}$ ions that are stored in the SR are released to the cytosol via the activated RyR1. The $\mathrm{Ca}^{2+}$ ions in the cytosol bind to troponin $\mathrm{C}$, which activates a series of contractile proteins to induce contraction in skeletal muscle fibers. Interestingly, during the skeletal EC coupling, DHPRs serve as activating ligands for RyR1 (2-5). Accordingly, Ca ${ }^{2+}$ entry via DHPRs is not a necessary factor for skeletal EC coupling, although $\mathrm{Ca}^{2+}$ entry via DHPR does exist, which is one of the critical differences between skeletal and cardiac EC coupling processes. Skeletal EC coupling is an outside-in signal from the DHPRs on t-tubule membranes to the internal RyR1 on the SR membrane and is regarded as an orthograde signal compared with other recently discovered signals.

A totally different protein is required to remove and recycle the $\mathrm{Ca}^{2+}$ ions from the cytosol during skeletal muscle relaxation - a reverse action to skeletal muscle contraction. During relaxation of the skeletal muscle, sarcoplasmic/ endoplasmic reticulum $\mathrm{Ca}^{2+}$-ATPase 1a (SERCA1a, a $\mathrm{Ca}^{2+}$ pump on the SR membrane) uptakes cytosolic $\mathrm{Ca}^{2+}$ ions into the SR in order to reduce the cytosolic $\mathrm{Ca}^{2+}$ level to that at rest as well as to refill the SR with $\mathrm{Ca}^{2+}$ ions $(3,23)$. SERCA1a is the major isoform in adults (24). Calsequestrin (CSQ), a luminal protein of the $\mathrm{SR}$, has a $\mathrm{Ca}^{2+}$-buffering ability that prevents the SR from swelling or bursting by osmotic pressure due to the high $\mathrm{Ca}^{2+}$ concentrations in the SR following skeletal muscle relaxation (i.e., at rest) (25).

\section{EMERGING PROTEINS RELATED TO SKELETAL EC COUPLING}

Modern physiologists who study skeletal muscle have steadily searched for and discovered new proteins with exclusive functions in skeletal muscle and have established their interactive mechanisms with EC coupling-mediating proteins. The triad junction supports an efficient arrangement of the EC coupling-mediating proteins on the SR and t-tubule membranes, which allows for the rapid and frequent delivery of $\mathrm{Ca}^{2+}$, depending on the purpose and on the appropriate retention of $\mathrm{Ca}^{2+}$ in skeletal muscle fibers $(3,4)$. Junctophilin 1 (JP1, also known as mitsugumin 72), JP2, or mitsugumin 29 (MG29) contributes to the formation and maintenance of the triad junction (3, 4, 19-22, 26).

The activity of the EC coupling-mediating proteins is regulated by other proteins. STIM1 on the SR membrane negatively regulates $\mathrm{Ca}^{2+}$ release from the SR through a direct interaction of its C-terminus with DHPRs (27). The activity of RyR1 is increased by the binding of the FK506-binding protein 12 (FKBP12) or JP1 (28-32). Triadin on the SR membrane interacts with RyR1 via its KEKE motifs and is required for typical RyR1 activity (33-35). SERCA1a activity is regulated, either by MG53 or STIM1: MG53 binds to SERCA1a via its TRIM-PRY domain and attenuates SERCA1a activity (36);
STIM1 binds to SERCA1a via its C-terminal portion, and is required for the full activity of SERCA1a (37). On the other hand, there are several proteins that do not directly bind to the EC coupling-mediating proteins but regulate the gain of EC coupling. Canonical-type transient receptor potential cation channel type 3 (TRPC3) is required to sustain high $\mathrm{Ca}^{2+}$ levels in the cytosol for the full gain of EC coupling $(3,38)$. For other proteins or factors that regulate skeletal EC coupling, either directly or indirectly, refer to primary review articles by Endo et al. (1977) and Zucchi et al. (1997) $(2,5)$.

\section{BREAKING FRESH GROUND IN SKELETAL MUSCLE RESEARCH}

Recent advances in the deep understanding of $\mathrm{Ca}^{2+}$ movements during skeletal muscle contraction and relaxation have established the involvement of extracellular $\mathrm{Ca}^{2+}$ in the various functions of skeletal muscles, including both contraction and relaxation; this has demonstrated the importance of $\mathrm{Ca}^{2+}$ entry from the extracellular space to the cytosol of skeletal muscle fibers. Extracellular $\mathrm{Ca}^{2+}$ entry into skeletal muscle fibers has gained significant attention over the past decade, although its existence was reported a long time ago (6-8).

SOCE is one of the methods for extracellular $\mathrm{Ca}^{2+}$ entry, and is also known as $\mathrm{Ca}^{2+}$ release-activated $\mathrm{Ca}^{2+}(\mathrm{CRAC})$ in mast cells $(6,7)$. The proteins required for SOCE have been identified over a 20-year period, since its discovery in the 1980s (6, 7). Orai1 (also known as CRACM1) works as the $\mathrm{Ca}^{2+}$ entry channel in plasma/sarcolemmal membranes during SOCE (14-18). The gating of Orai 1 depends on the state of the STIM1 located in the ER/SR membrane. STIM1 is a subunit-like protein of Orai1, rather than a regulatory protein (9-13), as described in the next section. STIM1 functions as a $\mathrm{Ca}^{2+}$ sensor for the amount of $\mathrm{Ca}^{2+}$ ions that are stored in the ER/SR. In skeletal muscle, SOCE contributes to the $\mathrm{Ca}^{2+}$ supply that is required for the maintenance of skeletal muscle contraction $(8,39)$. However, note that SOCE is not for the initiation of skeletal muscle contraction, as mentioned in the Introduction section; rather, SOCE is required for the fine-tuning of skeletal muscle functions, particularly for events taking place over a long period or with repetition. Furthermore, SOCE is believed to be one of the common tools that mediate various cellular events, including the progression of diseases, as well as physiological functions in skeletal muscle $(8,39)$.

\section{GENERAL OVERVIEW OF SOCE}

Generally speaking, $\mathrm{Ca}^{2+}$ depletion of the ER is the initiation signal that evokes SOCE. The $\mathrm{Ca}^{2+}$ depletion is sensed by the $\mathrm{N}$-terminus of STIM1, which induces the dimerization of STIM1s and the relocation of dimeric STIM1s to the ER membrane near the plasma membrane (10 to $25 \mathrm{~nm}$ gap 
between the two membranes) $(40,41)$. At the same time, some portions of the ER are rearranged towards the plasma membrane where the dimeric STIM1s relocate (42). It remains unclear, however, what initiates the ER rearrangement and STIM1 relocation, and in what order. In response to the ER rearrangement and STIM1 relocation, monomeric Orai1 on the plasma membrane simultaneously oligomerizes to form a hexameric and functional $\mathrm{Ca}^{2+}$ channel and relocates to the plasma membrane near the ER membrane containing the dimeric STIM1s; this allows for the formation of heteromeric oligomers that are composed of STIM1s and Orai1s (called 'puncta') (43). The formation of puncta allows an extracellular $\mathrm{Ca}^{2+}$ entry through Orai $1(44,45)$. The entire process of SOCE starting from $\mathrm{ER} \mathrm{Ca}^{2+}$ depletion to extracellular $\mathrm{Ca}^{2+}$ entry takes several seconds, depending on the types of cells (46). Two independent research teams have reported on the interaction of the domain of STIM1 to Orai 1 in the puncta: the CRAC activation domain (CAD) and the STIM1-Orai activating region (SOAR) (44, 45). CAD and SOAR overlap with one another and are referred to as CAD/SOAR, which is highly conserved and binds directly to the $\mathrm{N}$ - and C-termini of Orai 1 .

\section{SOCE IN THE SKELETAL MUSCLE}

In skeletal SOCE, the SR and t-tubule membranes substitute for the ER and plasma membranes, respectively, and Orai1 and STIM1 are also the main proteins that are responsible for skeletal SOCE (47-49). Interestingly, skeletal SOCE has exclusive properties that compare to properties in other types of cells or tissues. Orai 1 and STIM1 in skeletal muscle form puncta even during resting periods (i.e., puncta formations without the $\mathrm{Ca}^{2+}$ depletion from the SR) (Fig. 1A) (26, 27, 39). As mentioned in the Introduction section, skeletal muscle fibers have a specific membrane structure that is referred to as the triad junction. Closely juxtaposed t-tubule and SR membranes in the triad junction allow skeletal muscle to skip both the rearrangement of the SR membrane near the t-tubule membrane and the relocation of STIM1s or Orai1s to the rearranged SR or t-tubule membranes during skeletal SOCE. The formation of pre-puncta occurs during the development of skeletal muscle fibers (i.e., myogenesis) and during terminal differentiation, which is a process whereby skeletal myoblasts fuse and form multi-nucleated mature myotubes; myoblasts are the proliferative form of satellite cells, and these are also referred to as skeletal muscle stem cells $(26,27,39)$. The pre-puncta reside in an almost-ready-to-go state, and the conformational changes of Orai 1 and/or STIM1 seem to be required in order to evoke skeletal SOCE $(26,27,50)$. An advantage of the pre-puncta formation in the skeletal SOCE is faster kinetics (an immediate and rapid delivery of extracellular $\mathrm{Ca}^{2+}$ to the cytosol during the skeletal SOCE). The skeletal SOCE occurs in less than a second i.e., millisecond activation, which is significantly faster than in other types of cells that can require up to several seconds $(39,49,51)$. While skeletal SOCE is fast, it is relatively slower than classical $\mathrm{Ca}^{2+}$ movements in skeletal muscle fibers, such as intracellular
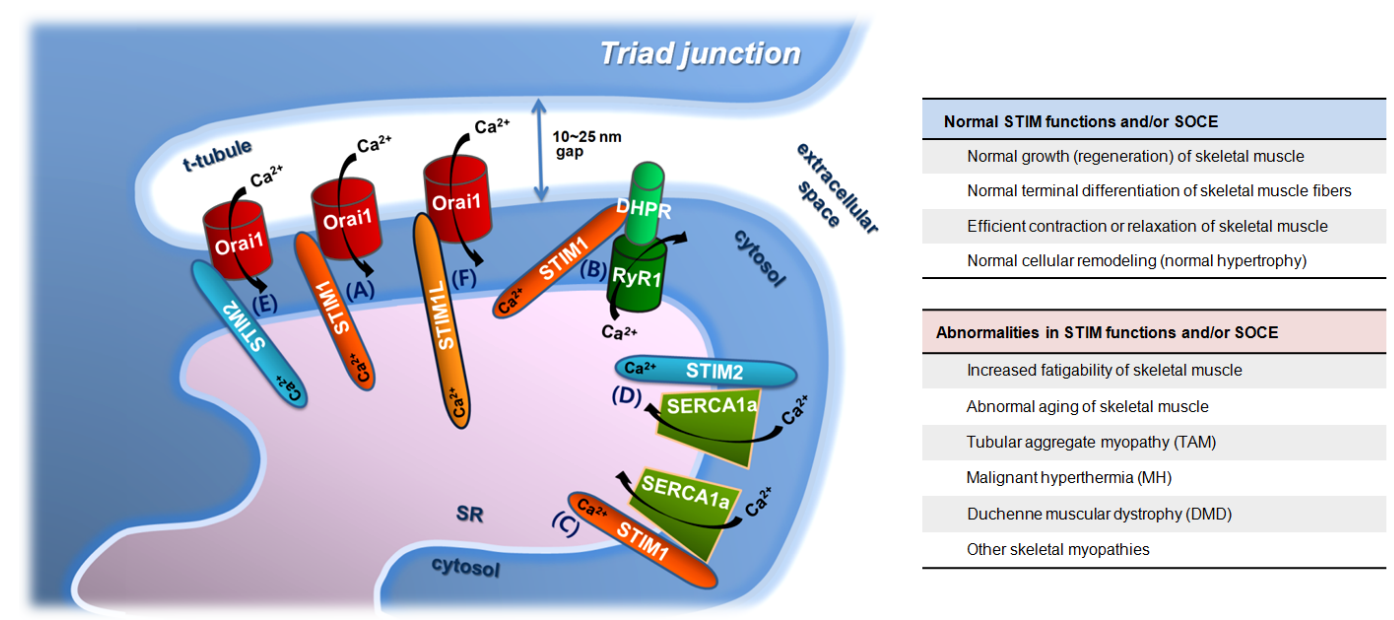

Fig. 1. $\mathrm{Ca}^{2+}$ movements involving STIMs and/or skeletal SOCE in skeletal muscle, and proteins that either regulate or are otherwise related to the $\mathrm{Ca}^{2+}$ movements. A triad junction with proteins that either regulate or are related to STIMs and skeletal SOCE is depicted, and the involvement of STIM proteins in the $\mathrm{Ca}^{2+}$ movements of skeletal muscle fibers is presented (A to F). DHPR, dihydropyridine receptors; RyR1, ryanodine receptor 1; STIM1, stromal interaction molecule 1; STIM2, stromal interaction molecule 2; STIM1L, a long form of STIM1; SERCA1a, sarcoplasmic/endoplasmic reticulum $\mathrm{Ca}^{2+}$-ATPase 1a; SR, sarcoplasmic reticulum; and, t-tubule, transverse-tubule. The direction of $\mathrm{Ca}^{2+}$ movements via the proteins is presented by black arrows. STIMs and/or skeletal SOCE participate in various skeletal muscle functions, ranging from short to long and from physiological to pathophysiological phenomena (summarized in the right-hand panel). Proteins that contribute to the formation and maintenance of the triad junction (JP1, JP2, or MG29) are not depicted, despite their significance. 
$\mathrm{Ca}^{2+}$ release to the cytosol during contraction or $\mathrm{Ca}^{2+}$ uptake to the SR during relaxation.

The SR in skeletal muscle fibers is subdivided by location (5). Junctional SR is also referred to terminal cisternae and participates in the formation of the triad junction, which is where STIM1 in other types of cells exist during SOCE. Longitudinal SR is not juxtaposed with t-tubule membranes. STIM1 in the skeletal muscle fibers is found in both types of the SR (39). The existence of graded SOCE, which is also referred to as delayed SOCE, has been reported in skeletal muscle, and STIM1 in the longitudinal SR could be responsible for the graded SOCE (52-54). Therefore, STIM1-mediated SOCE in skeletal muscle follows different time-courses according to the location of STIM1 in the SR, which could contribute to various functions involving the skeletal SOCE, with a delayed onset or an extended period.

Skeletal SOCE is one of the retrograde signals in terms of the working direction $\left(\mathrm{Ca}^{2+}\right.$ depletion from the SR is a signal from the internal SR that triggers activation of Orai1 in the t-tubule membrane) $(42,55)$. Therefore, the skeletal SOCE fundamentally differs from the movement of $\mathrm{Ca}^{2+}$ during orthograde $\mathrm{EC}$ coupling in terms of both the working direction and the source of $\mathrm{Ca}^{2+}$

\section{DOMAINS OF STIM1}

STIM1 has a short intra-luminal $\mathrm{N}$-terminus (with a signal peptide, a canonical EF-hand, a hidden EF-hand (pseudo EF-hand), and a sterile $\alpha$-motif (SAM) domain), a single transmembrane domain, and a cytosolic C-terminus (with three coiled-coil (CC) domains, a CAD/SOAR, a di-arginine consensus site, and a lysine-rich domain) $(9,13,56)$. The signal peptide is composed of 22 amino acids and directs STIM1 to the ER, which amounts to an ER retention signal (12, 57, 58). The CC domains, the lysine-rich domain, and the di-arginine consensus site also contribute to ER retention (57, 58). The D76, D84, and E87 residues in the canonical EF-hand are critical for sensing the amount of $\mathrm{Ca}^{2+}$ in the ER $(11,27$, $59,60)$. The EF-hands and SAM domain are responsible for the self-oligomerization and the relocalization of STIM1 during SOCE $(61,62)$. The first CC domain (CC1) participates in the oligomerization of STIM1 at rest (63). CAD/SOAR is the binding site to the $\mathrm{N}$ - and C-termini of Orai1 $(44,45)$. The lysine-rich domain is responsible for the Orai1-independent plasma membrane targeting of STIM1 (44).

\section{STIMs IN THE SKELETAL MUSCLE}

The domain arrangement and the function of each domain of STIM1 in the skeletal muscle are the same as those of STIM1 found in other types of cells. STIM1 binds to CSQ in hetrologous expression systems and the binding interferes with STIM1 dimerizations, with the binding between STIM1 and Orai1, and with the skeletal SOCE (64-67). STIM1 plays important roles in $\mathrm{Ca}^{2+}$ release and uptake during skeletal muscle contraction and relaxation, as well as in skeletal SOCE. STIM1 binds to DHPR and attenuates DHPR activity, which reduces intracellular $\mathrm{Ca}^{2+}$ release in response to contractile stimuli by way of DHPR, such as membrane depolarization during the EC coupling (Fig. 1B) (27). STIM1 also binds to SERCA1a via its C-terminal portion (amino acids from 449 to 671) and maintains the full activity of SERCA1a at a high cytosolic $\mathrm{Ca}^{2+}$ level. One such example is during skeletal muscle relaxation following contraction (Fig. 1C) (37). Therefore, STIM1 functions as an all-around player in $\mathrm{Ca}^{2+}$ movements in the skeletal muscle. It acts a faithful guardian of SR $\mathrm{Ca}^{2+}$ storage by sensing SR $\mathrm{Ca}^{2+}$ depletion during skeletal SOCE (Fig. 1A) and an attenuator of DHPR activity during skeletal muscle contraction (Fig. 1B). It also balances SERCA1a activity during skeletal muscle contraction and relaxation (Figs. $1 \mathrm{C}$ and 1D; for this, see the next paragraph).

In addition to the role of STIM1 in a single event, such as skeletal SOCE, it is also involved in long-term events such as terminal differentiation as well as neonatal muscle growth (68, 69). STIM1 expression has been detected in myoblasts before terminal differentiation. It gradually increases initially during the terminal differentiation until differentiation day 2 and is maintained during further differentiation days after a small decrease $(27,39)$. These changes in the expression level of STIM1 accompany the enhancement of skeletal SOCE during terminal differentiation $(27,39,70)$. STIM2 is also required for terminal differentiation $(71,72)$. STIM1L, in conjunction with TRPC1 and TRPC4, participates in the formation of larger myotubes during terminal differentiation (73).

STIM2 and STIM1L have been identified as homologues of STIM1 $(13,52,74)$. The physiological roles of the homologues are not as well defined as those for STIM1. STIM2 plays a role in SOCE in mild $\mathrm{Ca}^{2+}$ depletion of the ER, and regulates basal $\mathrm{Ca}^{2+}$ levels in a storage-independent manner in heterologous expression systems $(75,76)$. STIM1 and STIM2 are functionally redundant to some degree in skeletal muscle, such as in the case of positive controls in SOCE (Fig. 1E). Either STIM1 or STIM2 can correct most of the impact of STIM2- and STIM1silencing in skeletal SOCE and in terminal differentiation (71, $72,77)$. STIM2 acts similarly to STIM1 i.e., it also binds to SERCA1a via a C-terminal portion (amino acids from 453 to 729) (Fig. 1D) and contributes to intracellular $\mathrm{Ca}^{2+}$ distribution between the cytosol and the SR. However, the regulation of SERCA1a activity by STIM2 is in opposition to that by STIM1, and attenuation of SERCA1a activity by STIM2 is the reason STIMs work as balancers of SERCA1a activity (77). Thus, STIM1 and STIM2 function as fine-tuners that regulate SERCA1a activity in skeletal muscle fibers. Overall, the roles of STIM2 in skeletal muscle bear similarities and also differences to those of STIM1. This functional redundancy makes it difficult to discriminate the roles of STIM1 from those of STIM2. STIM1L is an alternatively spliced, longer variation of STIM1 $(47,52)$. It is abundantly expressed in skeletal muscle, 
but much less so in non-excitable cells $(47,52)$. STIM1L generates faster and repetitive skeletal SOCE through formation of permanent puncta (Fig. 1F) (52).

Taken together, STIMs and skeletal SOCE in the skeletal muscle play various roles in the integration of signals during growth, terminal differentiation, contraction and relaxation of skeletal muscle. These functions rely on spatial, temporal, and additional resources to utilize STIMs and skeletal SOCE.

\section{DOMAIN STRUCTURES IN STIM1}

Crystallographers have examined each domain of human STIM1, but not that of full-length STIM1. The 3-dimensional (3D) structure of SOAR that is capable of activating Orai 1 shows four helixes (78). Dimeric SOARs are necessary to activate Orai 1 (78). The CC1 domain has an adjacent inhibitory helix $(\mathrm{CC} 1-\mathrm{IH}$, amino acids from 237 to 340), and CC1-IH show a long helix and contributes to the oligomerization of STIM1 (79). The binding of IH to SOAR induces an inactive STIM1 dimer $(78,79)$. The CC1 and CC2 regions homodimerize in an antiparallel fashion and form a U-shaped structure (80). A trimer of the dimers of CC1 and CC2, which results in a hexameric STIM1 homomer, activates a hexameric Orai1 with a central ion-permeating pore $(41,81,82)$.

The canonical EF-hand is paired with a hidden EF-hand, and the EF-hand pair is then composed of ten short helixes that mediate mutually hydrophobic interactions between the EF-hand pair and the SAM domain (EF-SAM) (56). $\mathrm{Ca}^{2+}$ depletion makes the EF-SAM unstable by exposing several hydrophobic residues, and the unstable EF-SAM oligomerizes at the luminal portion of the SR to be energetically favorable. The 3D structure of the EF-SAM in STIM2 is not much different from that in STIM1, except for an expanded non-polar cleft (62). This suggests the possibility that other domains of STIM2 are not much different from those of STIM1, although the 3D structure of STIM2 remains unknown.

It is interesting that the 3D-structure of Orai1 differs markedly from that of other ion channels, and the functional Orai channel of Drosophila melanogaster exists as a hexameric assembly (a tetramer is common in other channels) $(81,82)$.

\section{SKELETAL MUSCLE FATIGUE OR AGING THAT INVOLVES SKELETAL SOCE}

The involvement of STIM1 and/or SOCE in relatively longer-term events of the skeletal muscle, such as fatigue and aging, is briefly mentioned in this section. Correlations between skeletal muscle fatigue and skeletal SOCE have been reported. Skeletal muscle fibers from transgenic mice with dominant-negative Orai1 display a lack of skeletal SOCE and an increased susceptibility to skeletal muscle fatigue (50). MG29-deficient mice also show an increased susceptibility to skeletal muscle fatigue due to abnormal triad junctions and severe dysfunctions in skeletal SOCE (83-85). Skeletal muscle fibers from sarcalumenin-deficient mice show increases in skeletal SOCE and fatigue resistance (86). Transgenic mice with sarcolipin are more resistant to skeletal muscle fatigue, and skeletal muscle fibers from these mice show an increase in skeletal SOCE (87). Exercising mice show extra junctions that are composed of the SR and t-tubule membranes containing Orai1 and STIM1 (88). The mice with the extra junction show increased resistance to skeletal muscle fatigue, and SOCE inhibitors abolish the increased resistance to skeletal muscle fatigue. Therefore, SOCE could be one of the fatigue-regulating factors in skeletal muscle.

Skeletal muscle fibers from aged mice (26-27 months corresponding to old age in humans) show severe reductions in skeletal SOCE (89). However, the reduction in the skeletal SOCE is not the result of a change in either STIM1 or Orai 1 expression levels. In accordance with this fact, the expression levels of neither STIM1 nor Orai1 are changed during skeletal muscle aging in humans, mice, or flies (90). It is possible that changes in the functional properties of STIM1 and Orai1, possibly in conjunction with the regulatory proteins, could govern the aging processes of skeletal muscle. Unlike cardiac muscle, hypertrophy in skeletal muscle is related to healthy phenomena such as muscle growth, repair, or regeneration (i.e., cellular remodeling) $(91,92)$. Healthy hypertrophy in skeletal muscle involves increases in skeletal SOCE $(69,93)$. For both a broader and a deeper understanding of the fundamental aspects of aging in skeletal muscle, readers are encouraged to refer to a study by Zahn et al. (90), where the transcriptional profiling of aging in human, mouse, and fly muscle is well defined using common aging signature sets.

\section{SKELETAL MUSCLE DISEASES THAT INVOLVE STIM1 AND/OR SKELETAL SOCE}

Skeletal muscle diseases are extremely complex issues in terms of their causes, onsets, levels of progress, symptoms, and prognoses. In this section, the involvement of STIM1 and/or skeletal SOCE in skeletal muscle diseases are briefly mentioned. However, as far as we could ascertain, there are no reports on the involvement of either STIM2 or STIM1L in skeletal muscle diseases.

Patients with a loss-of-function mutation of STIM1, E136X, show severe combined immunodeficiency (SCID) and congenital myopathies such as non-progressive muscular hypotonia due to lack of skeletal SOCE (94). Patients with another type of STIM1 mutation, R429C, also show muscular hypotonia (95). STIM1 deficiency in mice results in a perinatally lethal condition, which is characterized by a failure to show skeletal SOCE, a significant reduction in body weight, skeletal-muscle hypotonia, and a significant increase in susceptibility to skeletal muscle fatigue (39). STIM1 transgenic mice show a significant increase in skeletal SOCE, as observed in dystrophic muscle fibers (96). These reports suggest that STIM1 in skeletal muscle is involved in diseases such as skeletal 
muscle dystrophies as well as in physiological phenomena such as growth, terminal differentiation, and contraction and relaxation of skeletal muscle.

Tubular aggregate myopathy (TAM), a genetically heterogeneous disease, is characterized by tubular aggregates within skeletal muscle fibers (97). These tubular aggregates are among the secondary features that are representative markers of various human myopathies (97). Mutations in STIM1 have been found in patients with TAM (N80T, G81D, L96V, F108I, H109R, I115F, or I484R) (98-101). In particular, patients with one of four STIM1 mutations in the EF-hands or a region near the hidden EF-hand $(\mathrm{H} 72 \mathrm{Q}$, D84G, H109N, or H109R that are constitutively active forms of STIM1) also show atrophy of skeletal muscles and progressive muscle weaknesses involving excessive levels of skeletal SOCE and a significantly higher cytosolic $\mathrm{Ca}^{2+}$ level (102). Therefore, STIM1 is among the genetic causes of TAM.

When volatile anesthetics are administered to patients with malignant hyperthermia $(\mathrm{MH})$, a pharmacogenetic skeletal muscle disorder, it can lead to life-threatening skeletal muscle contracture: An increase in body temperature due to the uncontrolled elevation of cytosolic $\mathrm{Ca}^{2+}$ levels is mediated by uncontrolled activation of RyR1, which ultimately causes sudden death $(103,104)$. A sustained skeletal SOCE also contributes to the maintained and uncontrolled elevation of cytosolic $\mathrm{Ca}^{2+}$ levels in patients with $\mathrm{MH}$ (105). In addition, $\mathrm{MH}$-like symptoms have been observed in the knock-out mice of either CSQ1 or CSQ2 or the knock-in mice of RyR1 mutation, wherein the increase in body temperature is related to an increase in skeletal SOCE $(67,106-108)$. It is possible that skeletal SOCE could also be involved in the progression of central core disease (CCD involves progressive muscle weakness), because patients with CCD are also at high risk for the development of $\mathrm{MH}$ (109).

Duchenne muscular dystrophy (DMD) is a lethal form of skeletal muscular dystrophy that is characterized by progressive wasting of the skeletal muscle (110). The enhancement of skeletal SOCE in the skeletal muscle fibers of $m d x$ mice (a mouse model of DMD) is accompanied by significant increases in STIM1 and Orai1 expression (111-113). Surprisingly, undifferentiated myoblasts from $m d x$ mice have also shown enhanced SOCE, with a significant increase in STIM1 expression (114). Skeletal muscle fibers from transgenic mice with muscle-specific STIM1 show characteristics of DMD such as a significant increase in skeletal SOCE (96). Therefore, abnormalities in STIM1 expression and skeletal SOCE are causes of DMD pathogenesis.

The involvement of STIM1 and/or SOCE in skeletal muscle diseases, by way of proteins such as TRPC 3 and MG53, have also been reported in the literature (115-121). In addition, other possible factors that could contribute to skeletal muscle diseases have also been examined in several articles (54, 122-124). As shown in other studies, Orai 1 is also significantly related to skeletal muscle diseases as well as physiological functions (125-128).

\section{CONCLUDING REMARKS}

Extracellular $\mathrm{Ca}^{2+}$ entry into skeletal muscle via SOCE, particularly STIM-mediated SOCE, is the focus of this mini-review. STIMs and/or STIM-mediated skeletal SOCE participate in various skeletal muscle functions, ranging from short to long-term functions, and from physiological to pathophysiological phenomena, such as contraction and relaxation, growth, terminal differentiation, cellular remodeling, fatigue, aging, and diseases. Thus, it seems that STIMs always verify what the skeletal muscle is doing and tunes the various functions of skeletal muscle with the greatest of care.

The involvement of STIMs in both healthy and diseased states of skeletal muscles implies that these proteins may have dual roles. Unfortunately, despite all the evidence and the importance of the involvement of STIMs in various skeletal muscle functions, the extent of the extracellular $\mathrm{Ca}^{2+}$ contribution via STIM-mediating skeletal SOCE versus the intracellular $\mathrm{Ca}^{2+}$ contribution to skeletal muscle functioning has not been well addressed. Studies on the relative extent of extracellular $\mathrm{Ca}^{2+}$ contributions via STIMs to skeletal muscle functions could provide answers as to how the dual roles of these protein functions in the body could be utilized.

\section{ACKNOWLEDGEMENTS}

This work was supported by the Mid-career Researcher Program through National Research Foundation of Korea grants funded by the Korean government (No. NRF-2017R1 A2B4005924 to E.H.L.)

\section{CONFLICTS OF INTEREST}

The authors have no conflicting interests.

\section{REFERENCES}

1. Marieb EN and Hoehn K (2010) Human anatomy \& physiology, 8th ed, Benjamin Cummings, San Francisco

2. Zucchi R and Ronca-Testoni S (1997) The sarcoplasmic reticulum $\mathrm{Ca}^{2+}$ channel/ryanodine receptor: modulation by endogenous effectors, drugs and disease states. Pharmacol Rev 49, 1-51

3. Lee EH (2010) $\mathrm{Ca}^{2+}$ channels and skeletal muscle diseases. Prog Biophys Mol Biol 103, 35-43

4. Lee EH, Kim DH and Allen PD (2006) Interplay between intra- and extracellular calcium ions. Mol Cells 21, 315-329

5. Endo M (1977) Calcium release from the sarcoplasmic reticulum. Physiol Rev 57, 71-108

6. Putney JW Jr. (1986) A model for receptor-regulated calcium entry. Cell Calcium 7, 1-12

7. Hoth M and Penner R (1992) Depletion of intracellular calcium stores activates a calcium current in mast cells. 
Nature 355, 353-356

8. Kurebayashi N and Ogawa Y (2001) Depletion of $\mathrm{Ca}^{2+}$ in the sarcoplasmic reticulum stimulates $\mathrm{Ca}^{2+}$ entry into mouse skeletal muscle fibres. J Physiol 533, 185-199

9. Zhang SL, Yu Y, Roos J et al (2005) STIM1 is a $\mathrm{Ca}^{2+}$ sensor that activates CRAC channels and migrates from the $\mathrm{Ca}^{2+}$ store to the plasma membrane. Nature 437, 902-905

10. Roos J, DiGregorio PJ, Yeromin AV et al (2005) STIM1, an essential and conserved component of store-operated $\mathrm{Ca}^{2+}$ channel function. J Cell Biol 169, 435-445

11. Liou J, Kim ML, Heo WD et al (2005) STIM is a $\mathrm{Ca}^{2+}$ sensor essential for $\mathrm{Ca}^{2+}$-store-depletion-triggered $\mathrm{Ca}^{2+}$ influx. Curr Biol 15, 1235-1241

12. Parker NJ, Begley CG, Smith PJ and Fox RM (1996) Molecular cloning of a novel human gene (D11S4896E) at chromosomal region 11p15.5. Genomics 37, 253-256

13. Williams RT, Manji SS, Parker NJ et al (2001) Identification and characterization of the STIM (stromal interaction molecule) gene family: coding for a novel class of transmembrane proteins. Biochem J 357, 673-685

14. Yeromin AV, Zhang SL, Jiang W, Yu Y, Safrina $O$ and Cahalan MD (2006) Molecular identification of the CRAC channel by altered ion selectivity in a mutant of Orai. Nature 443, 226-229

15. Prakriya M, Feske S, Gwack Y, Srikanth S, Rao A and Hogan PG (2006) Orai1 is an essential pore subunit of the CRAC channel. Nature 443, 230-233

16. Vig $M$, Beck A, Billingsley JM et al (2006) CRACM1 multimers form the ion-selective pore of the CRAC channel. Curr Biol 16, 2073-2079

17. Feske S, Gwack Y, Prakriya M et al (2006) A mutation in Orai1 causes immune deficiency by abrogating CRAC channel function. Nature 441, 179-185

18. Vig M, Peinelt $C$, Beck A et al (2006) CRACM1 is a plasma membrane protein essential for store-operated $\mathrm{Ca}^{2+}$ entry. Science 312, 1220-1223

19. Ito K, Komazaki S, Sasamoto K et al (2001) Deficiency of triad junction and contraction in mutant skeletal muscle lacking junctophilin type 1. J Cell Biol 154, 1059-1067

20. Woo JS, Cho CH, Lee KJ, Kim DH, Ma J and Lee EH (2012) Hypertrophy in skeletal myotubes induced by junctophilin-2 mutant, $\mathrm{Y} 141 \mathrm{H}$, involves an increase in store-operated $\mathrm{Ca}^{2+}$ entry via Orai1. J Biol Chem 287, 14336-14348

21. Komazaki S, Nishi M, Takeshima $\mathrm{H}$ and Nakamura $\mathrm{H}$ (2001) Abnormal formation of sarcoplasmic reticulum networks and triads during early development of skeletal muscle cells in mitsugumin29-deficient mice. Dev Growth Differ 43, 717-723

22. Nishi M, Komazaki S, Kurebayashi $\mathrm{N}$ et al (1999) Abnormal features in skeletal muscle from mice lacking mitsugumin29. J Cell Biol 147, 1473-1480

23. Shamoo AE and MacLennan DH (1974) $\mathrm{A} \mathrm{Ca}^{++}$. dependent and -selective ionophore as part of the $\mathrm{Ca}^{++}$ plus $\mathrm{Mg}^{++}$-dependent adenosinetriphosphatase of sarcoplasmic reticulum. Proc Natl Acad Sci U S A 71, 35223526

24. Brandl CJ, deLeon S, Martin DR and MacLennan DH
(1987) Adult forms of the $\mathrm{Ca}^{2+}$ ATPase of sarcoplasmic reticulum. Expression in developing skeletal muscle. J Biol Chem 262, 3768-3774

25. Murphy RM, Larkins NT, Mollica JP, Beard NA and Lamb GD (2009) Calsequestrin content and SERCA determine normal and maximal $\mathrm{Ca}^{2+}$ storage levels in sarcoplasmic reticulum of fast- and slow-twitch fibres of rat. J Physiol 587, 443-460

26. Woo JS, Cho $\mathrm{CH}$, Lee $\mathrm{KJ}$, Kim DH, Ma J and Lee EH (2012) Hypertrophy in skeletal myotubes induced by junctophilin-2 mutant, $\mathrm{Y} 141 \mathrm{H}$, involves an increase in store-operated $\mathrm{Ca}^{2+}$ entry via Orai1. J Biol Chem 287, 14336-14348

27. Lee KJ, Woo JS, Hwang JH et al (2013) STIM1 negatively regulates $\mathrm{Ca}^{2+}$ release from the sarcoplasmic reticulum in skeletal myotubes. Biochem J 453, 187-200

28. Jayaraman T, Brillantes AM, Timerman AP et al (1992) FK506 binding protein associated with the calcium release channel (ryanodine receptor). J Biol Chem 267, 9474-9477

29. Timerman AP, Ogunbumni E, Freund E, Wiederrecht G, Marks AR and Fleischer S (1993) The calcium release channel of sarcoplasmic reticulum is modulated by FK-506-binding protein. Dissociation and reconstitution of FKBP-12 to the calcium release channel of skeletal muscle sarcoplasmic reticulum. J Biol Chem 268, 22992-22999

30. Avila G, Lee EH, Perez CF, Allen PD and Dirksen RT (2003) FKBP12 binding to RyR1 modulates excitationcontraction coupling in mouse skeletal myotubes. J Biol Chem 278, 22600-22608

31. Lee EH, Rho SH, Kwon SJ, Eom SH, Allen PD and Kim $\mathrm{DH}$ (2004) $\mathrm{N}$-terminal region of FKBP12 is essential for binding to the skeletal ryanodine receptor. J Biol Chem 279, 26481-26488

32. Phimister AJ, Lango J, Lee EH et al (2007) Conformationdependent stability of junctophilin 1 (JP1) and ryanodine receptor type 1 (RyR1) channel complex is mediated by their hyper-reactive thiols. J Biol Chem 282, 8667-8677

33. Lee EH, Song DW, Lee JM, Meissner G, Allen PD and Kim DH (2006) Occurrence of atypical $\mathrm{Ca}^{2+}$ transients in triadin-binding deficient-RYR1 mutants. Biochem Biophys Res Commun 351, 909-914

34. Lee JM, Rho SH, Shin DW et al (2004) Negatively charged amino acids within the intraluminal loop of ryanodine receptor are involved in the interaction with triadin. J Biol Chem 279, 6994-7000

35. Guo W and Campbell KP (1995) Association of triadin with the ryanodine receptor and calsequestrin in the lumen of the sarcoplasmic reticulum. J Biol Chem 270, 9027-9030

36. Lee KJ, Park CS, Woo JS, Kim DH, Ma J and Lee EH (2012) Mitsugumin 53 attenuates the activity of sarcoplasmic reticulum $\mathrm{Ca}^{2+}$-ATPase 1a (SERCA1a) in skeletal muscle. Biochem Biophys Res Commun 428, 383-388

37. Lee KJ, Hyun C, Woo JS, Park CS, Kim DH and Lee EH (2014) Stromal interaction molecule 1 (STIM1) regulates sarcoplasmic/endoplasmic reticulum $\mathrm{Ca}^{2+}$-ATPase $1 \mathrm{a}$ (SERCA1a) in skeletal muscle. Pflugers Arch 466, 987-1001 
38. Lee EH, Cherednichenko G, Pessah IN and Allen PD (2006) Functional coupling between TRPC3 and RyR1 regulates the expressions of key triadic proteins. J Biol Chem 281, 10042-10048

39. Stiber J, Hawkins A, Zhang ZS et al (2008) STIM1 signalling controls store-operated calcium entry required for development and contractile function in skeletal muscle. Nat Cell Biol 10, 688-697

40. Luik RM, Wang B, Prakriya $M, W u$ MM and Lewis RS (2008) Oligomerization of STIM1 couples ER calcium depletion to CRAC channel activation. Nature 454, 538-542

41. Zhou Y, Wang X, Wang X et al (2015) STIM1 dimers undergo unimolecular coupling to activate Orai1 channels. Nat Commun 6, 8395

42. Luik RM, Wu MM, Buchanan J and Lewis RS (2006) The elementary unit of store-operated $\mathrm{Ca}^{2+}$ entry: local activation of CRAC channels by STIM1 at ER-plasma membrane junctions. J Cell Biol 174, 815-825

43. Penna A, Demuro A, Yeromin AV et al (2008) The CRAC channel consists of a tetramer formed by Stim-induced dimerization of Orai dimers. Nature 456, 116-120

44. Park CY, Hoover PJ, Mullins FM et al (2009) STIM1 clusters and activates CRAC channels via direct binding of a cytosolic domain to Orai1. Cell 136, 876-890

45. Yuan JP, Zeng W, Dorwart MR, Choi YJ, Worley PF and Muallem S (2009) SOAR and the polybasic STIM1 domains gate and regulate Orai channels. Nat Cell Biol $11,337-343$

46. Lewis RS (2007) The molecular choreography of a store-operated calcium channel. Nature 446, 284-287

47. Launikonis BS, Murphy RM and Edwards JN (2010) Toward the roles of store-operated $\mathrm{Ca}^{2+}$ entry in skeletal muscle. Pflugers Arch 460, 813-823

48. Ma J and Pan Z (2003) Retrograde activation of storeoperated calcium channel. Cell Calcium 33, 375-384

49. Launikonis BS and Rios E (2007) Store-operated $\mathrm{Ca}^{2+}$ entry during intracellular $\mathrm{Ca}^{2+}$ release in mammalian skeletal muscle. J Physiol 583, 81-97

50. Wei-Lapierre L, Carrell EM, Boncompagni S, Protasi F and Dirksen RT (2013) Orai1-dependent calcium entry promotes skeletal muscle growth and limits fatigue. Nat Commun 4, 2805

51. Edwards JN, Murphy RM, Cully TR, von Wegner F, Friedrich O and Launikonis BS (2010) Ultra-rapid activation and deactivation of store-operated $\mathrm{Ca}^{2+}$ entry in skeletal muscle. Cell Calcium 47, 458-467

52. Darbellay B, Arnaudeau S, Bader CR, Konig S and Bernheim L (2011) STIM1L is a new actin-binding splice variant involved in fast repetitive $\mathrm{Ca}^{2+}$ release. J Cell Biol 194, 335-346

53. Hirata $Y$, Brotto $M$, Weisleder $N$ et al (2006) Uncoupling store-operated $\mathrm{Ca}^{2+}$ entry and altered $\mathrm{Ca}^{2+}$ release from sarcoplasmic reticulum through silencing of junctophilin genes. Biophys J 90, 4418-4427

54. Zhao X, Weisleder N, Han X et al (2006) Azumolene inhibits a component of store-operated calcium entry coupled to the skeletal muscle ryanodine receptor. J Biol Chem 281, 33477-33486

55. Huang GN, Zeng W, Kim JY et al (2006) STIM1 carboxyl-terminus activates native SOC, I(crac) and TRPC1 channels. Nat Cell Biol 8, 1003-1010

56. Stathopulos PB, Zheng L, Li GY, Plevin MJ and Ikura M (2008) Structural and mechanistic insights into STIM1mediated initiation of store-operated calcium entry. Cell $135,110-122$

57. Ercan E, Chung SH, Bhardwaj R and Seedorf M (2012) Di-arginine signals and the K-rich domain retain the $\mathrm{Ca}^{2+}$ sensor STIM1 in the endoplasmic reticulum. Traffic $13,992-1003$

58. Saitoh N, Oritani K, Saito K et al (2011) Identification of functional domains and novel binding partners of STIM proteins. J Cell Biochem 112, 147-156

59. Spassova MA, Soboloff J, He LP, Xu W, Dziadek MA and Gill DL (2006) STIM1 has a plasma membrane role in the activation of store-operated $\mathrm{Ca}^{2+}$ channels. Proc Natl Acad Sci U S A 103, 4040-4045

60. Grosse J, Braun A, Varga-Szabo D et al (2007) An EF hand mutation in Stim1 causes premature platelet activation and bleeding in mice. J Clin Invest 117, 35403550

61. Stathopulos PB, Zheng L and Ikura M (2009) Stromal interaction molecule (STIM) 1 and STIM2 calcium sensing regions exhibit distinct unfolding and oligomerization kinetics. J Biol Chem 284, 728-732

62. Zheng L, Stathopulos PB, Schindl R, Li GY, Romanin C and Ikura M (2011) Auto-inhibitory role of the EF-SAM domain of STIM proteins in store-operated calcium entry. Proc Natl Acad Sci U S A 108, 1337-1342

63. Covington ED, Wu MM and Lewis RS (2010) Essential role for the CRAC activation domain in store-dependent oligomerization of STIM1. Mol Biol Cell 21, 1897-1907

64. Wang L, Zhang L, Li S et al (2015) Retrograde regulation of STIM1-Orai1 interaction and store-operated $\mathrm{Ca}^{2+}$ entry by calsequestrin. Sci Rep 5, 11349

65. Zhang L, Wang L, Li S, Xue J and Luo D (2016) Calsequestrin-1 regulates store-operated $\mathrm{Ca}^{2+}$ entry by inhibiting STIM1 aggregation. Cell Physiol Biochem 38, 2183-2193

66. Shin DW, Pan Z, Kim EK et al (2003) A retrograde signal from calsequestrin for the regulation of store-operated $\mathrm{Ca}^{2+}$ entry in skeletal muscle. J Biol Chem 278, 3286-3292

67. Zhao X, Min CK, Ko JK et al (2010) Increased storeoperated $\mathrm{Ca}^{2+}$ entry in skeletal muscle with reduced calsequestrin-1 expression. Biophys J 99, 1556-1564

68. Lee HJ, Bae GU, Leem YE et al (2012) Phosphorylation of Stim 1 at serine 575 via netrin-2/Cdo-activated ERK1/2 is critical for the promyogenic function of Stim1. Mol Biol Cell 23, 1376-1387

69. Li T, Finch EA, Graham V et al (2012) STIM1-Ca ${ }^{2+}$ signaling is required for the hypertrophic growth of skeletal muscle in mice. Mol Cell Biol 32, 3009-3017

70. Darbellay B, Arnaudeau S, Konig S et al (2009) STIM1and Orai1-dependent store-operated calcium entry regulates human myoblast differentiation. J Biol Chem 284, 5370-5380

71. Darbellay B, Arnaudeau S, Ceroni D, Bader CR, Konig S and Bernheim L (2010) Human muscle economy myoblast differentiation and excitation-contraction coupling use the same molecular partners, STIM1 and STIM2. J Biol 
Chem 285, 22437-22447

72. Phuong TTT and Kang TM (2015) Stromal interaction molecule 2 regulates $\mathrm{C} 2 \mathrm{C} 12$ myoblast differentiation. Integr Med Res 4, 242-248

73. Antigny F, Sabourin J, Sauc S, Bernheim L, Koenig S and Frieden M (2017) TRPC1 and TRPC4 channels functionally interact with STIM1L to promote myogenesis and maintain fast repetitive $\mathrm{Ca}^{2+}$ release in human myotubes. Biochim Biophys Acta 1864, 806-813

74. Hoth M and Niemeyer BA (2013) The neglected CRAC proteins: Orai2, Orai3, and STIM2. Curr Top Membr 71, 237-271

75. Brandman O, Liou J, Park WS and Meyer T (2007) STIM2 is a feedback regulator that stabilizes basal cytosolic and endoplasmic reticulum $\mathrm{Ca}^{2+}$ levels. Cell $131,1327-1339$

76. Wang X, Wang $Y$, Zhou $Y$ et al (2014) Distinct Orai-coupling domains in STIM1 and STIM2 define the Orai-activating site. Nat Commun 5, 3183

77. Oh MR, Lee KJ, Huang M et al (2017) STIM2 regulates both intracellular $\mathrm{Ca}^{2+}$ distribution and $\mathrm{Ca}^{2+}$ movement in skeletal myotubes. Sci Rep 7, 17936

78. Yang X, Jin H, Cai X, Li S and Shen Y (2012) Structural and mechanistic insights into the activation of Stromal interaction molecule 1 (STIM1). Proc Natl Acad Sci U S A 109, 5657-5662

79. Cui B, Yang X, Li S et al (2013) The inhibitory helix controls the intramolecular conformational switching of the C-terminus of STIM1. PLoS One 8, e74735

80. Stathopulos PB, Schindl R, Fahrner M et al (2013) STIM1/Orai1 coiled-coil interplay in the regulation of store-operated calcium entry. Nat Commun 4, 2963

81. Hou X, Pedi L, Diver MM and Long SB (2012) Crystal structure of the calcium release-activated calcium channel Orai. Science 338, 1308-1313

82. Rothberg BS, Wang Y and Gill DL (2013) Orai channel pore properties and gating by STIM: implications from the Orai crystal structure. Sci Signal 6, pe9

83. Pan Z, Yang D, Nagaraj RY et al (2002) Dysfunction of store-operated calcium channel in muscle cells lacking mg29. Nat Cell Biol 4, 379-383

84. Brotto MA, Nagaraj RY, Brotto LS, Takeshima H, Ma JJ and Nosek TM (2004) Defective maintenance of intracellular $\mathrm{Ca}^{2+}$ homeostasis is linked to increased muscle fatigability in the MG29 null mice. Cell Res 14 , 373-378

85. Nagaraj RY, Nosek CM, Brotto MA et al (2000) Increased susceptibility to fatigue of slow- and fast-twitch muscles from mice lacking the MG29 gene. Physiol Genomics 4, 43-49

86. Zhao X, Yoshida M, Brotto $L$ et al (2005) Enhanced resistance to fatigue and altered calcium handling properties of sarcalumenin knockout mice. Physiol Genomics 23, 72-78

87. Sopariwala DH, Pant M, Shaikh SA et al (2015) Sarcolipin overexpression improves muscle energetics and reduces fatigue. J Appl Physiol (1985) 118, 1050-1058

88. Boncompagni S, Michelucci A, Pietrangelo L, Dirksen RT and Protasi F (2017) Exercise-dependent formation of new junctions that promote STIM1-Orai1 assembly in skeletal muscle. Sci Rep 7, 14286

89. Zhao X, Weisleder N, Thornton A et al (2008) Compromised store-operated $\mathrm{Ca}^{2+}$ entry in aged skeletal muscle. Aging Cell 7, 561-568

90. Zahn JM, Sonu R, Vogel H et al (2006) Transcriptional profiling of aging in human muscle reveals a common aging signature. PLoS Genet 2, e115

91. Almada AE and Wagers AJ (2016) Molecular circuitry of stem cell fate in skeletal muscle regeneration, ageing and disease. Nat Rev Mol Cell Biol 17, 267-279

92. Berchtold MW, Brinkmeier $\mathrm{H}$ and Muntener M (2000) Calcium ion in skeletal muscle: its crucial role for muscle function, plasticity, and disease. Physiol Rev 80, 1215-1265

93. Woo JS, Hwang JH, Ko JK et al (2010) S165F mutation of junctophilin 2 affects $\mathrm{Ca}^{2+}$ signalling in skeletal muscle. Biochem J 427, 125-134

94. Picard C, McCarl CA, Papolos A et al (2009) STIM1 mutation associated with a syndrome of immunodeficiency and autoimmunity. N Engl J Med 360, 1971-1980

95. Fuchs S, Rensing-Ehl A, Speckmann C et al (2012) Antiviral and regulatory $T$ cell immunity in a patient with stromal interaction molecule 1 deficiency. J Immunol $188,1523-1533$

96. Goonasekera SA, Davis J, Kwong JQ et al (2014) Enhanced $\mathrm{Ca}^{2+}$ influx from STIM1-Orai1 induces muscle pathology in mouse models of muscular dystrophy. Hum Mol Genet 23, 3706-3715

97. Morgan-Hughes JA (1998) Tubular aggregates in skeletal muscle: their functional significance and mechanisms of pathogenesis. Curr Opin Neurol 11, 439-442

98. Tasca G, D'Amico A, Monforte M et al (2015) Muscle imaging in patients with tubular aggregate myopathy caused by mutations in STIM1. Neuromuscul Disord 25, 898-903

99. Okuma H, Saito F, Mitsui J et al (2016) Tubular aggregate myopathy caused by a novel mutation in the cytoplasmic domain of STIM1. Neurol Genet 2, e50

100. Walter MC, Rossius M, Zitzelsberger M et al (2015) 50 years to diagnosis: Autosomal dominant tubular aggregate myopathy caused by a novel STIM1 mutation. Neuromuscul Disord 25, 577-584

101. Bohm J, Chevessier F, Koch C et al (2014) Clinical, histological and genetic characterisation of patients with tubular aggregate myopathy caused by mutations in STIM1. J Med Genet 51, 824-833

102. Bohm J, Chevessier F, Maues De Paula A et al (2013) Constitutive activation of the calcium sensor STIM1 causes tubular-aggregate myopathy. Am J Hum Genet 92, 271-278

103. MacLennan DH and Phillips MS (1992) Malignant hyperthermia. Science 256, 789-794

104. Nelson TE (2002) Malignant hyperthermia: a pharmacogenetic disease of $\mathrm{Ca}^{++}$regulating proteins. Curr Mol Med 2, 347-369

105. Duke AM, Hopkins PM, Calaghan SC, Halsall JP and Steele DS (2010) Store-operated $\mathrm{Ca}^{2+}$ entry in malignant hyperthermia-susceptible human skeletal muscle. J Biol Chem 285, 25645-25653 
106. Dainese $M$, Quarta $M$, Lyfenko AD et al (2009) Anesthetic- and heat-induced sudden death in calsequestrin-1-knockout mice. FASEB J 23, 1710-1720

107. Yarotskyy V, Protasi F and Dirksen RT (2013) Accelerated activation of SOCE current in myotubes from two mouse models of anesthetic- and heat-induced sudden death. PLoS One 8, e77633

108. Chelu MG, Goonasekera SA, Durham WJ et al (2006) Heat- and anesthesia-induced malignant hyperthermia in an RyR1 knock-in mouse. FASEB J 20, 329-330

109. Jungbluth H (2007) Central core disease. Orphanet J Rare Dis 2, 25

110. Ervasti JM and Campbell KP (1993) Dystrophin-associated glycoproteins: their possible roles in the pathogenesis of Duchenne muscular dystrophy. Mol Cell Biol Hum Dis Ser 3, 139-166

111. Edwards JN, Friedrich O, Cully TR, von Wegner F, Murphy RM and Launikonis BS (2010) Upregulation of store-operated $\mathrm{Ca}^{2+}$ entry in dystrophic $\mathrm{mdx}$ mouse muscle. Am J Physiol Cell Physiol 299, C42-50

112. Boittin FX, Petermann O, Hirn C et al (2006) $\mathrm{Ca}^{2+}$ independent phospholipase A2 enhances store-operated $\mathrm{Ca}^{2+}$ entry in dystrophic skeletal muscle fibers. J Cell Sci $119,3733-3742$

113. Zhao X, Moloughney JG, Zhang S, Komazaki S and Weisleder N (2012) Orai1 mediates exacerbated $\mathrm{Ca}^{2+}$ entry in dystrophic skeletal muscle. PLoS One 7, e49862

114. Onopiuk M, Brutkowski W, Young C et al (2015) Store-operated calcium entry contributes to abnormal $\mathrm{Ca}^{2+}$ signalling in dystrophic $\mathrm{mdx}$ mouse myoblasts. Arch Biochem Biophys 569, 1-9

115. Takamori M (2008) Autoantibodies against TRPC3 and ryanodine receptor in myasthenia gravis. J Neuroimmunol 200, 142-144

116. Zhang BT, Yeung SS, Cheung KK, Chai ZY and Yeung EW (2014) Adaptive responses of TRPC1 and TRPC3 during skeletal muscle atrophy and regrowth. Muscle Nerve 49, 691-699

117. Cai C, Masumiya H, Weisleder N et al (2009) MG53 nucleates assembly of cell membrane repair machinery. Nat Cell Biol 11, 56-64

118. He B, Tang RH, Weisleder N et al (2012) Enhancing muscle membrane repair by gene delivery of MG53 ameliorates muscular dystrophy and heart failure in delta-Sarcoglycan-deficient hamsters. Mol Ther 20, 727-735

119. Weisleder N, Takizawa N, Lin P et al (2012) Recombinant MG53 protein modulates therapeutic cell membrane repair in treatment of muscular dystrophy. Sci Transl Med 4, 139ra185

120. Cai C, Weisleder N, Ko JK et al (2009) Membrane repair defects in muscular dystrophy are linked to altered interaction between MG53, caveolin-3, and dysferlin. J Biol Chem 284, 15894-15902

121. Ahn MK, Lee KJ, Cai $C$ et al (2016) Mitsugumin 53 regulates extracellular $\mathrm{Ca}^{2+}$ entry and intracellular $\mathrm{Ca}^{2+}$ release via Orai1 and RyR1 in skeletal muscle. Sci Rep 6, 36909

122. Vandebrouck C, Martin D, Colson-Van Schoor $M$, Debaix H and Gailly P (2002) Involvement of TRPC in the abnormal calcium influx observed in dystrophic $(\mathrm{mdx})$ mouse skeletal muscle fibers. J Cell Biol 158, 1089-1096

123. Yang T, Allen PD, Pessah IN and Lopez JR (2007) Enhanced excitation-coupled calcium entry in myotubes is associated with expression of RyR1 malignant hyperthermia mutations. J Biol Chem 282, 37471-37478

124. Cherednichenko G, Ward CW, Feng W et al (2008) Enhanced excitation-coupled calcium entry in myotubes expressing malignant hyperthermia mutation R163C is attenuated by dantrolene. Mol Pharmacol 73, 1203-1212

125. Cho CH, Woo JS, Perez CF and Lee EH (2017) A focus on extracellular $\mathrm{Ca}^{2+}$ entry into skeletal muscle. Exp Mol Med 49, e378

126. Feske S (2010) CRAC channelopathies. Pflugers Arch 460, 417-435

127. Lee EH, Woo JS, Hwang JH, Park JH and Cho CH (2013) Angiopoietin 1 enhances the proliferation and differentiation of skeletal myoblasts. J Cell Physiol 228, 1038-1044

128. Woo JS, Cho CH, Kim DH and Lee EH (2010) TRPC3 cation channel plays an important role in proliferation and differentiation of skeletal muscle myoblasts. Exp Mol Med 42, 614-627 